\title{
Deformation Simulation of Laser Welding 304L Stainless Steel Thin Plate on the Bulging Process for Manufacturing New ice Machine*
}

\author{
by Ying WANG**, Jian $\mathrm{LUO}^{* * *}$, Fei LI ${ }^{* *}$ and Song FENG**
}

\begin{abstract}
Hydroforming is a manufacturing process that uses a fluid medium to form a part by using high internal pressure. The bulging process is one of the important methods for manufacturing the serpentine flowing channel evaporation board for the ice machine. The serpentine flowing channel evaporation board is made by the laser welding, which is of a high and complicated nonlinear deformation characteristic in bulging. In this paper, one simple practical model is applied to simulate the deformation and check the reliability of welding joint on bulging process. The bulging key parameters--bulging pressure, size of flowing channels and loading path are determined and optimized in order to avoid the uneven bulging. The height change and thickness distribution of welding structure's bulging deformation are discussed respectively.
\end{abstract}

Key Words: Bulging, Numerical Simulation, Stainless Steel), Laser Welding

\section{Introduction}

Hydroforming is a manufacturing process that uses a fluid medium to form a part by using high internal pressure ${ }^{1)}$. Grey et al. $^{2)}$ is the first to report on hydroforming of seamless copper fittings with T-branches. Fuchs's achievement is the first publication on hydrobulging research for metal forming, which described the feasibility of this technology used in actual production ${ }^{3)}$. Then many researchers have carried out experimental and theoretical research on the instability phenomena occurred in bulging process ${ }^{4)}$. Recent the new methods and characteristics of hydroforming, especially sheet hydroforming and tube hydroforming are all developed very quickly ${ }^{5)}$.

Many researchers have studied on numerical simulation on the hydroforming process using axisymmetric or non-symmetrical mathematical models and FEM ${ }^{6,7)}$. In addition, because the load line of bulging seriously affects the hydroforming, so many the research works have involved the optimization of loading path. However, there is still a lack of practical detailed research works about welding structure's hydroforming process.

Laser welding, which is a high power density and low heat input process offers clarifications to a wide range of problems usually encountered with the normal welding methods. Laser welding was performed by robots equipped with laser-focusing heads for close processing. Conventional welding processes such resistant spot and plasma welding are gradually replaced by laser welding technology within the automotive industry.

In this study, the deformation of the serpentine flowing channel evaporation board is studied on the bulging process,

\footnotetext{
${ }^{*}$ Received: 2012.11.28

${ }^{* *}$ Master Student, State Key Lab of Mechanical Transmission, Chongqing University, Chongqing, China

${ }^{* * *}$ Professor, State Key Lab of Mechanical Transmission, Chongqing University, Chongqing, China
}

which is made up of two stainless steel thin plates welded together by the laser welding method ${ }^{8,9)}$. The designs of welding structures and serpentine flowing channel are considered. The bulging procedures are optimized by using FEM analysis and physical simulation experiments.

\section{FEM Model}

Hydroforming control equation is described as follows:

$$
M \ddot{x}+c \dot{x}+K x=f_{e x t}+f_{e}
$$

Where ${ }^{x}$ is the nodal displacement vector, $\dot{x}$ is the node velocity vector, $\ddot{x}$ is the node acceleration vector, $M$ is mass matrix, $f_{e x t}$ is the external Force vector, $f_{e}$ is the contact force vector, ${ }^{c}$ is damping matrix, and $K$ is the overall stiffness matrix .

The three-dimensional FEM model of evaporation board of serpentine flowing channel and honeycomb flowing channel are shown in Fig.1 respectively, the welding seams or welding points are marked as these fuscous frames.

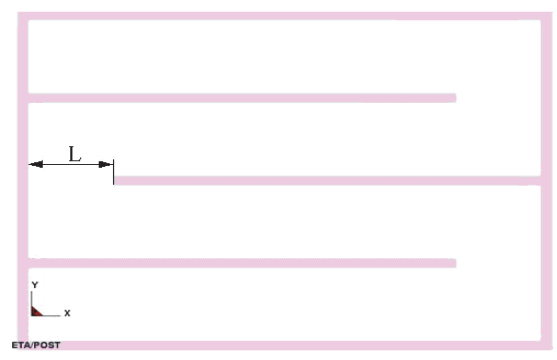

(a) Welding structure of serpentine flowing channel

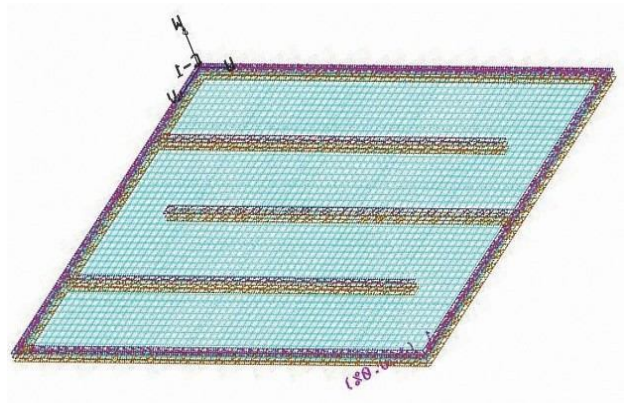

(b) Mesh of serpentine flowing channel 


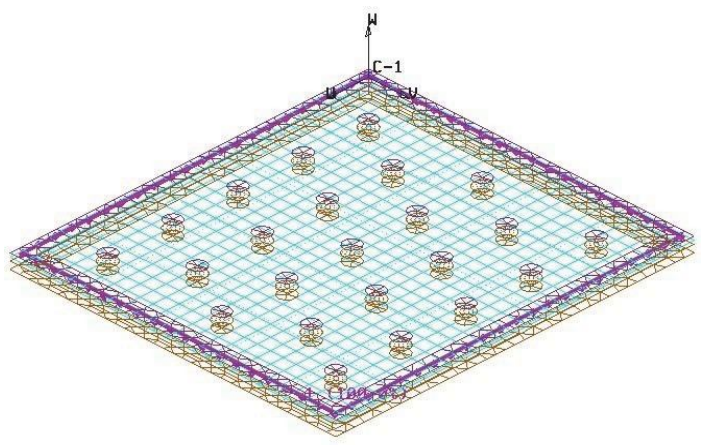

(c) Mesh of honeycomb flowing channel

Fig.1 Schematic diagram of 3D FEM model

In this paper, the theory of incremental plastic deformation, membrane theories and Hill's theory are applied in the liquid bulge forming of welding workpieces. The details of plastic deformation theory are cited as the reference 5-7. The material is $304 \mathrm{~L}$ stainless steel, tensile strength is $550 \mathrm{MPa}$, yield strength is $200 \mathrm{MPa}$, hardness is $187 \mathrm{HB}$, cross- section contraction rate is $55 \%$, the hardening index is 0.43 .

\section{Result and discussion}

\subsection{Determination of bulging pressure}

The simulation results at different bulging pressures are shown in Fig.2. The Forming Limit Diagram (FLD) is shown in Fig.3.

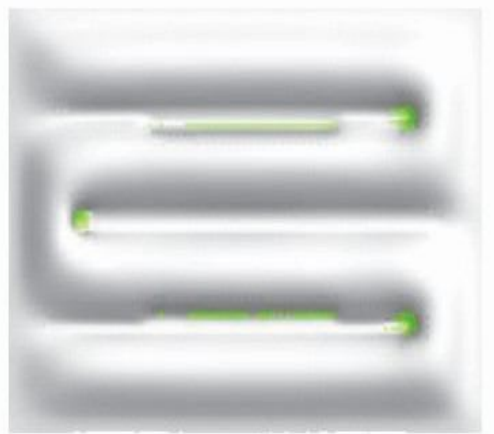

(a) $1 \mathrm{MPa}$

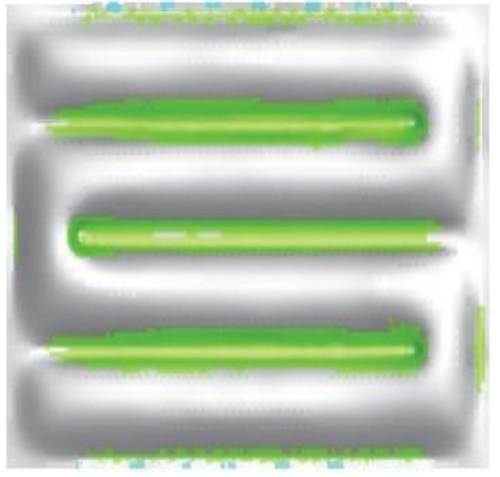

(b) $2 \mathrm{MPa}$
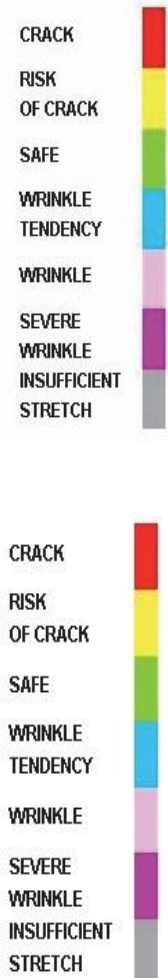

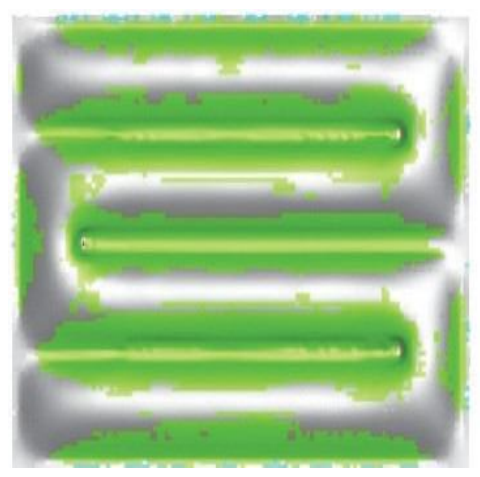

CRACK

RISK

OF CRACK

SAFE

WRINKLE

TENDENCY

WRINKLE

SEVERE

MRINKL

INSUFFICIENT

STRETCH
Fig.2 Deformations at different bulging pressures

Three Cases of bulging pressures are used in the serpentine flowing channel evaporation board, such as $1 \mathrm{MPa}, 2 \mathrm{MPa}$ and $3 \mathrm{MPa}$. In the Fig.2 and Fig.3, when the bulging pressure is $1 \mathrm{MPa}$, the serpentine flowing channel evaporation board has no wrinkling, cracking and other defects, and the formation lies in the safe range of Forming Limit Diagram (FLD). But the whole board takes place incomplete deformation, most of serpentine flowing channel zone is not sufficient. Rebound phenomenon after removal of the bulging pressure is more prominent. When the bulging pressure is $3 \mathrm{MPa}$, the deformation of metal sheet is overfull, the edges of metal sheet has a slightly wrinkled, the end of welding seam has a cracked trend, the formation lies in the damage range of FLD, which do not meet the technical requirements of evaporation plate.

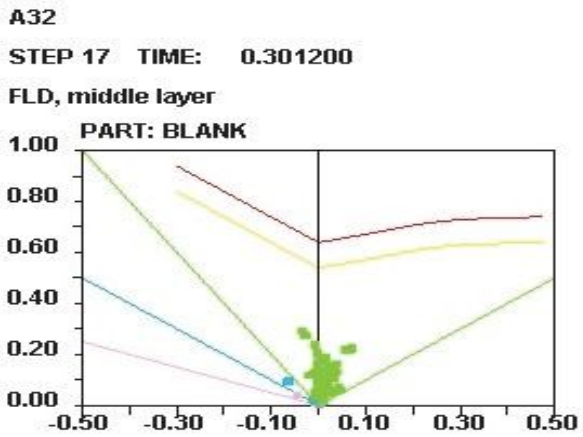

(a) $2 \mathrm{MPa}$

P3

STEP 17 TIMIE: 0.301200

FLD, middle layer

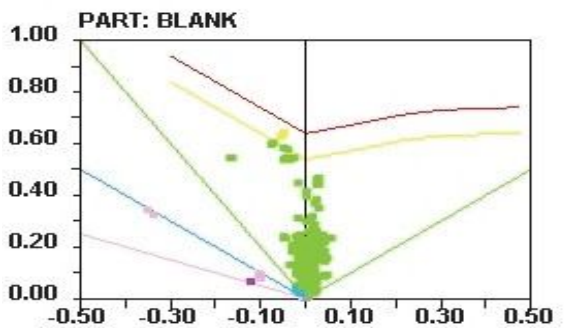

(b) $3 \mathrm{MPa}$

Fig.3 FLD at different bulging pressures 
In this Case, the $2 \mathrm{MPa}$ bulging pressure is determined as a reasonable bulging pressure by the comparing of the forming results.

\subsection{Effect of welding structure}

Three width Cases of the serpentine flowing channel evaporation board are used in this paper, this is marked as " $\mathrm{L}$ " shown in Fig.1(a). The results at different flowing channel width $(\mathrm{L}=30 \mathrm{~mm}, \mathrm{~L}=32 \mathrm{~mm}$ and $\mathrm{L}=35 \mathrm{~mm})$ are shown in Fig. 4 respectively.

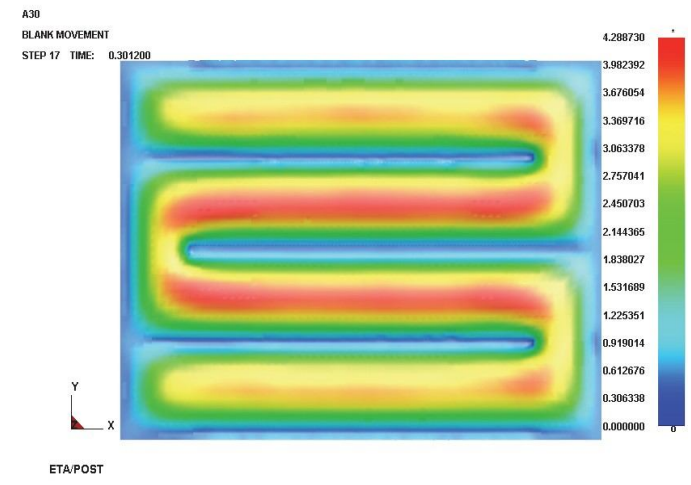

(a) $\mathrm{L}=30 \mathrm{~mm}$

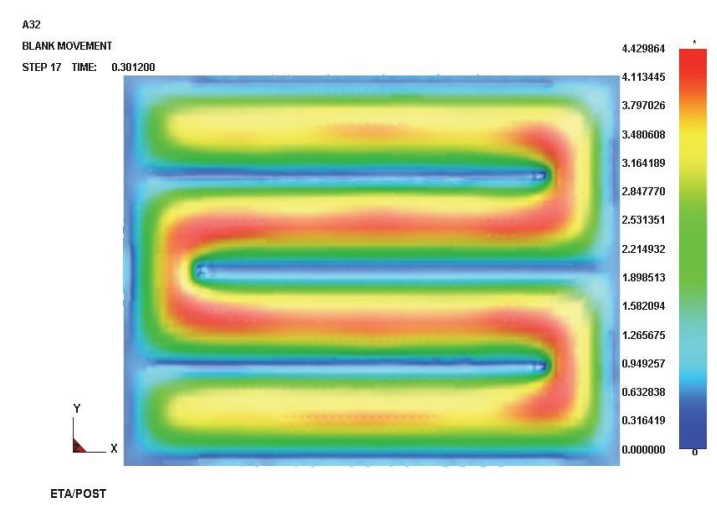

(b) $\mathrm{L}=32 \mathrm{~mm}$

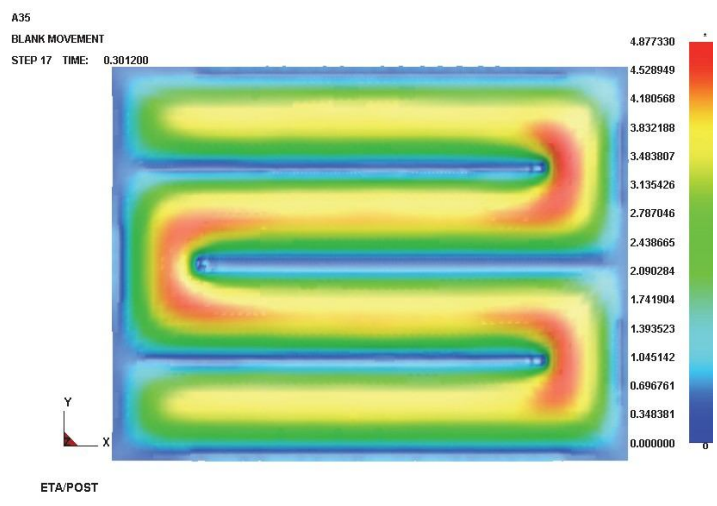

(c) $\mathrm{L}=35 \mathrm{~mm}$

Fig.4 Deformation at different flowing channel width
$32 \mathrm{~mm}$, the deformation shows that the height of whole board is uniform, special at the corner regions. The thickness distribution of deformation is more even than that of other Cases. Therefore, the suitable width of serpentine flowing channel value or welding structure is $32 \mathrm{~mm}$.

The stainless steel evaporator plate of honeycomb flowing channel is made by the laser spot welding and formed by liquid bulge forming method. This simulation process is same as that of the serpentine flowing channel. The sheet thickness is $10 \mathrm{~mm}$, the size of plate is $110 \mathrm{~mm} \times 100 \mathrm{~mm}$. the diameter of laser welding spot is $5 \mathrm{~mm}$, the distance between adjacent welding spot is $20 \mathrm{~mm}$. The bulging pressure is $5 \mathrm{MPa}$. The thickness distribution of honeycomb flowing channel evaporator plate is shown in Fig.5.

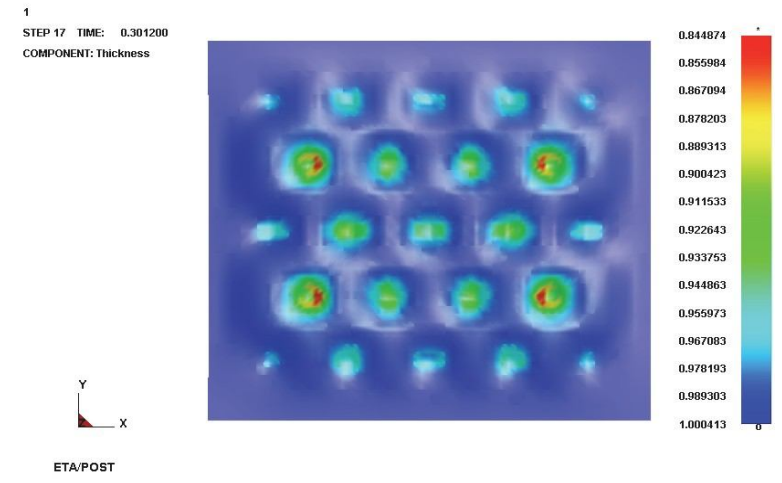

Fig.5 Thickness distribution of honeycomb flowing channel

The Fig.6 shows the forming of honeycomb flowing channel evaporator plate, when the bulging pressure is $2 \mathrm{MPa}$.

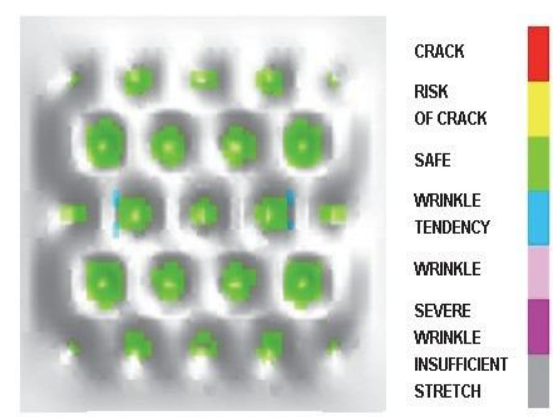

(a) Forming

STEP 17 TIME: 0.301200

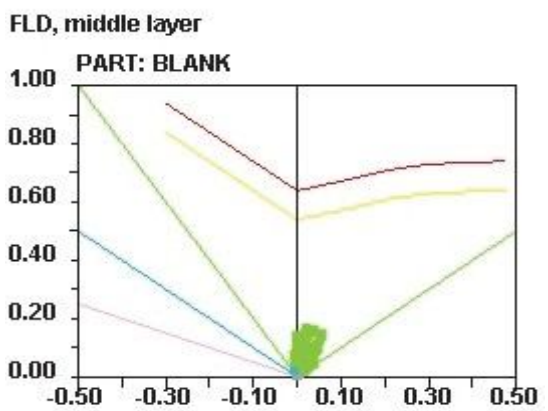

(b) FLD

Fig.6 Honeycomb flowing channel

In Fig.4, when the width of serpentine flowing channel is 
In the Fig.5, the thickness distribution of honeycomb flowing channel evaporator plate can be divided into two different regions: welding spot area and non-welding area. The welding spot and its vicinity are the most intense region of thickness variation. The thinnest part of evaporator plate occurs in this region, which is also the most dangerous zone (red part shown). The minimum value of thickness is $0.84 \mathrm{~mm}$. The thinning variation rate is $16 \%$ after the liquid bulge forming. The variation of thickness in the non-welded zone is very small, which is very safe.

In the Fig.6, when the bulging pressure and loading path are same as that of serpentine flowing channel (2MPa, Case I loading path, shown in Fig.7). The deformation is mainly concentrated in the welding spot. Most regional deformation is not sufficient, shown in silver-white part.

Compare with serpentine flowing channel, the honeycomb flowing channel evaporator plate needs greater hydraulic bulging pressure. Because many laser welding spots have a strong binding ability for the stainless steel plate.

\subsection{Effect of loading paths}

As shown in Fig.7, the bulging pressure increased from 0 to 2 $\mathrm{MPa}$, there have three Cases of loading path in bulging are used in this paper. The results of different loading paths in bulging are shown in Table 1. In the Fig.7, the maximum pressure is $2 \mathrm{MPa}$ and each loading time is same. The loading rate of bulging pressure decreased from Case I to Case III.

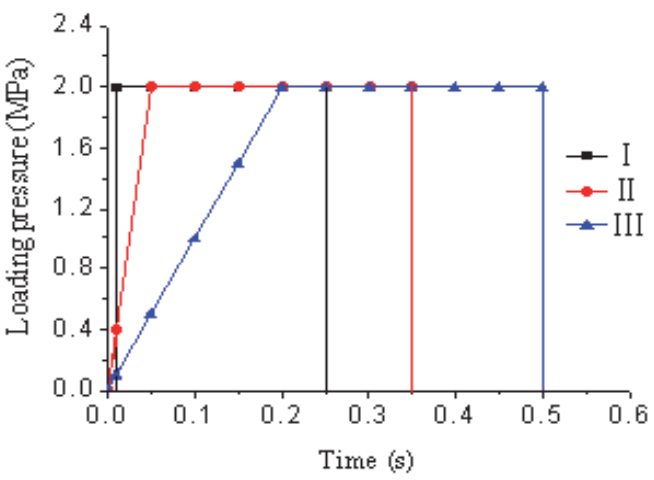

Fig.7 Loading paths in bulging

Table 1 Results of different loading paths

\begin{tabular}{lccc} 
Loading path & Case I & Case II & Case III \\
\hline $\begin{array}{l}\text { The Max height } \\
\text { of bulging (mm) }\end{array}$ & 4.42 & 2.95 & 2.61 \\
\hline $\begin{array}{l}\text { The Min size of } \\
\text { thickness (mm) }\end{array}$ & 0.75 & 0.97 & 0.96 \\
\hline
\end{tabular}

The Table 1 shows that the deformation is depended on the loading rate of bulging pressure seriously. The Case II is suitable loading path or loading rate of bulging pressure, because both the Max height of bulging and the Min. size of thickness are more reasonable than that of Case I and Case III.

\subsection{Experimental results and model check}

The experimental results and simulation results of the serpentine flowing channel evaporation board are compared and shown in the Fig. 8 .

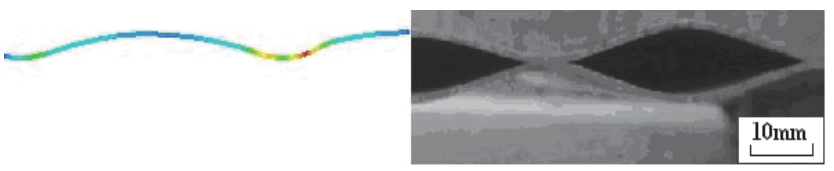

Fig.8 Comparison of simulation results and experimental results

The simulated cross-section shape of the serpentine flowing channel evaporation board is approximately to the practical serpentine flowing channel evaporation board after bulging. So the numerical simulation can be used to guide forming process in the paper.

\section{Conclusions}

The deformation of 304L stainless steel thin plate's welding structure on the bulging process is finished successfully for manufacturing the new ice machine. The height and thickness distribution of bulging deformation are depended on bulging pressure, loading rate or loading path, size of plate, and flowing channel structure seriously. When the flowing channel width is $32 \mathrm{~mm}$, bulging pressure is $2 \mathrm{MPa}$ and the loading path is Case II. The honeycomb flowing channel evaporator plate needs greater hydraulic bulging force than that of serpentine flowing channel evaporator plate. The bulging formation of the evaporation board is reasonable and reliable in this paper.

\section{Acknowledgements}

The authors appreciate the Fundamental Research Funds for the Central University (CDJRC11280002), Excellent Talents Project in Universities of Chongqing Municipal Education Commission (2010), Chongqing of P.R.China, and Fundamental Research Funds for State Key Lab of Mechanical Transmission ( SKLMT-ZZKT-2012MS08), Chongqing University, P.R.China.

\section{Reference}

1) K.I. Manabe and M. Amino: Effects of process parameters and material properties on deformation process in tube hydroforming, J. Mater. Process. Technol., 123 (2002), 285-291. 
2) Grey, J.E., Devereaux, A.P. and W.N. Parker: Apparatus for making wrought metal T's, US Patent 2,203,868 June (1939).

3) F.J Fuchs: Hydrostatic pressure: its role in metal forming. Mech. Eng., 88 (1966), 34-40.

4) S. Thiruvarudchelvan and W. Lewis: A note on hydroforming with constant fluid pressure, J. Mater. Process. Technol., 88 (1999) 51-56.

5) M.Koc and T.Altan: An overall review of the tube hydroforming (THF) technology, J. Mater. Process. Technol. 108(2001)384-393.

6) M. Imaninejad, G.subhash and A.Loukus: Experimental and numerical investigation of free-bulge formation during hydroforming of aluminum extrusions, J. Mater. Process. Technol., 147(2004) 247-254.
7) F. Djavanroodi, M. Gheisary and H. Zoghi-shal: Analytical and numerical analysis of free bulge tube hydroforming, American J Appl. Sci., 5(2008) 972-979.

8) Luo J, Zhang HW, Liu D, Wu ZJ and Yin DK: The welding deformation control of the cellular ice-maker plate in high-power CO2 laser welding condition, J. Chongqing Univ., 35(2012)11-17.

9) Luo J, Liu D, Yin DK, Yang JF, Zhang YC and T AKIYAMA: Influence of Welding Power to the Microstructure and Properties of 304 Stainless Steel Sheet Joints in CO2 Laser Welding, Rare Metal Mater. Eng., 40(2011)106-110. 